\title{
JSH Statement: Kyoto declaration on hypertension research in Asia
}

\author{
Masaaki Ito ${ }^{1}$ Toshihiko Ishimitsu ${ }^{2}$ Atsuhiro Ichihara ${ }^{3} \cdot$ Hiroshi Itoh $^{4} \cdot$ Mitsuru Ohishi $^{5} \cdot$ Takayoshi Ohkubo $^{6}$. \\ Hisashi Kai ${ }^{7} \cdot$ Naoki Kashihara $^{8} \cdot$ Kazuomi Kario $^{9} \cdot$ Shigeyuki Saitoh $^{10} \cdot$ Takuya Tsuchihashi $^{11} \cdot$ Satoko Nakamura $^{12}$. \\ Akira Nishiyama $^{13} \cdot$ Koichi Node $^{14} \cdot$ Naoyuki Hasebe $^{15} \cdot$ Jitsuo Higaki $^{16} \cdot$ Katsuyuki Miura $^{17} \cdot$ Hiromi Rakugi $^{18}$. \\ Kouichi Tamura ${ }^{19} \cdot$ Yuhei Kawano $^{20} \cdot$ Takashi Yatabe $^{21} \cdot$ Yusuke Ohya $^{22} \cdot$ Sadayoshi Ito $^{23}$
}

Received: 8 November 2018 / Accepted: 11 November 2018 / Published online: 7 January 2019

(c) The Japanese Society of Hypertension 2019

People in different parts of Asia share some common characteristics in terms of culture, customs, and ethnic factors that substantially affect the development, prevalence, and persistence of hypertension. We consider hypertension to be a condition that Asian countries should work closely together on to share information, and approaches should be created to reduce hypertension. As such, the Japanese Society of Hypertension (JSH) strives to make contributions so that biomedical researchers and clinical practitioners can learn from each other, share knowledge and experiences as well as discuss the latest research findings together in order to tackle common hypertension-related problems.

With these goals in mind, the JSH will (1) make its conferences more attractive to encourage the participation of researchers and clinicians from Asia and (2) establish a new fellowship system with the title of International Fellow of the Japanese Society of Hypertension (IFJSH) to honor dedicated researchers from Asia as well as international members in the field of hypertension.

The JSH wishes to serve as a medium for active discussion and fostering friendship. It is also our wish that
Asian researchers and practitioners become International Fellows of the Japanese Society of Hypertension and that these activities will promote international exchange and mutual understanding. Based on a deeper understanding of Asian hypertension through cooperative efforts, we aspire to generate novel solutions for this common goal.

Today, at the site of Annual Scientific Forum of Clinical Hypertension held by the Japanese Society of Hypertension in Kyoto, we pledge to make unwavering contributions to the development of hypertension clinical practice and high blood pressure research in Asia.

Acknowledgements We thank the members of the Working Group on the International Fellow of the Japanese Society of Hypertension (IFJSH), including Mari Ishida, Takashi Yokoo, and Midori Yatabe, for their help in developing this declaration.

\section{Compliance with ethical standards}

Conflict of interest The authors declare that they have no conflict of interest.

Kyoto Declaration on Hypertension Research in Asia was announced at the 7th Annual Scientific Forum of Clinical Hypertension of the JSH, which was held in Kyoto on May 19-20, 2018.

Masaaki Ito

mitoka@clin.medic.mie-u.ac.jp

Extended author information available on the last page of the article 


\section{Affiliations}

Masaaki Ito ${ }^{1} \cdot$ Toshihiko Ishimitsu $^{2} \cdot$ Atsuhiro Ichihara $^{3} \cdot$ Hiroshi Itoh $^{4} \cdot$ Mitsuru Ohishi $^{5} \cdot$ Takayoshi Ohkubo $^{6}$. Hisashi Kai $^{7}$ Naoki Kashihara ${ }^{8}$ Kazuomi Kario ${ }^{9} \cdot$ Shigeyuki Saitoh $^{10} \cdot$ Takuya Tsuchihashi $^{11} \cdot$ Satoko Nakamura $^{12}$. Akira Nishiyama ${ }^{13} \cdot$ Koichi Node $^{14} \cdot$ Naoyuki Hasebe $^{15} \cdot$ Jitsuo Higaki $^{16} \cdot$ Katsuyuki Miura $^{17} \cdot$ Hiromi Rakugi $^{18}$. Kouichi Tamura ${ }^{19} \cdot$ Yuhei Kawano ${ }^{20} \cdot$ Takashi Yatabe $^{21} \cdot$ Yusuke Ohya $^{22} \cdot$ Sadayoshi Ito ${ }^{23}$

1 Department of Cardiology and Nephrology, Mie University Graduate School of Medicine, Tsu, Japan

2 Department of Cardiology and Nephrology, Dokkyo Medical University Hospital, Mibu, Japan

3 Department of Endocrinology and Hypertension, Tokyo Women's Medical University, Tokyo, Japan

4 Department of Endocrinology, Metabolism and Nephrology, School of Medicine, Keio University, Tokyo, Japan

5 Department of Cardiovascular Medicine and Hypertension, Graduate School of Medicine and Dental Sciences, Kagoshima University, Kagoshima, Japan

6 Department of Hygiene and Public Health, Teikyo University School of Medicine, Tokyo, Japan

7 Department of Cardiology, Kurume University Medical Center, Kurume, Japan

8 Department of Nephrology and Hypertension, Kawasaki Medical School, Kurashiki, Japan

9 Division of Cardiovascular Medicine, Department of Medicine, Jichi Medical University School of Medicine, Shimotsuke, Japan

10 School of Health Sciences, Sapporo Medical University, Sapporo, Japan

11 Hypertension Center, Steel Memorial Yawata Hospital, Kitakyushu, Japan

12 Department of Nutritional Science for Well-being, Kansai University of Welfare Sciences, Kashiwara, Japan
13 Department of Pharmacology, Kagawa University Medical School, Kagawa, Japan

14 Department of Cardiovascular Medicine, Saga University, Saga, Japan

15 Division of Cardiology, Nephrology, Pulmonology and Neurology, Department of Internal Medicine, Asahikawa Medical University, Asahikawa, Japan

16 Minami-Matsuyama Hospital, Ehime University, Matsuyama, Japan

17 Department of Public Health, Shiga University of Medical Science, Otsu, Japan

18 Department of Geriatric and General Medicine, Osaka University Graduate School of Medicine, Suita, Japan

19 Department of Medical Science and Cardio-Renal Medicine, Yokohama City University Graduate School of Medicine, Yokohama, Japan

20 Department of Medical Technology, Teikyo University Fukuoka, Omuta, Japan

21 Yatabe \& Momii Law Office, Tokyo, Japan

22 Department of Cardiovascular Medicine, Nephrology and Neurology, University of the Ryukyus Graduate School of Medicine, Okinawa, Japan

23 Division of Nephrology, Endocrinology and Hypertension, Tohoku University Graduate School of Medicine, Sendai, Japan 